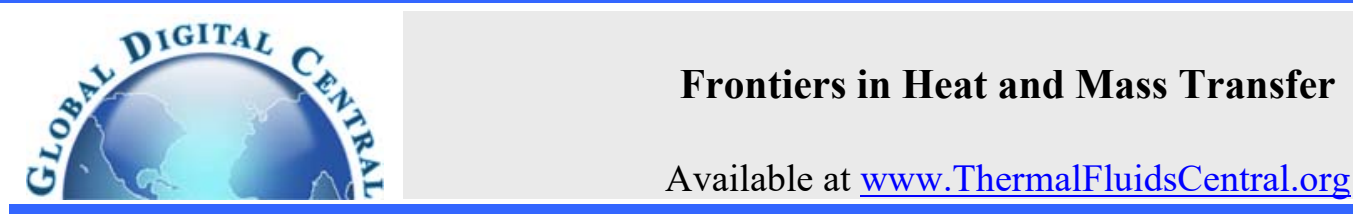

\title{
MHD VISCOUS CASSONFLUID FLOW IN THE PRESENCE OF A TEMPERATURE GRADIENT DEPENDENT HEAT SINK WITH PRESCRIBED HEAT AND MASS FLUX
}

\author{
S. Palaniammal ${ }^{1}$, K. Saritha ${ }^{2 *}$ \\ ${ }^{1}$ Department of Mathematics, Sri Krishna College of Technology, Coimbatore-642042, Tamil Nadu, India \\ ${ }^{2}$ Department of Mathematics, P. A. College of Engineering and Technology, Coimbatore-642002, Tamil Nadu, India
}

\begin{abstract}
This paper investigates heat and mass transfer of a MHD Casson fluid over a permeable stretching surface in the presence of a temperature gradient heat sink. The effects of viscous dissipation, thermal radiation and chemical reaction are also taken into the consideration. The relevant similarity transformations are used to reduce the governing equations into a system of nonlinear ordinary differential equations and then solved analytically. The influence of various physical parameters on the velocity, temperature, concentration, skin friction coefficient, Nusselt and Sherwood numbers are investigated. The numerical results of skin friction factor, Nusselt and Sherwood number are compared with the previously published data for special cases and are found to be in good agreement.
\end{abstract}

Keywords: Porous surface, Cassonfluid; Radiation, Chemical reaction, Heat and Mass flux.

\section{INTRODUCTION}

Newtonian fluids, in turbulence and multi-phase flows of the fluid dynamics for the past three centuries, have come a long way. They exhibit shear stress, which is proportional to the rate of shear at constant temperature and pressure and these constant of proportionality is known as the viscosity. The viscosity, in common, decreases with temperature and increases with pressure. Most of the liquid on the other hand, for instance, in the case of gases, the viscosity increases both with the temperature and pressure. However, for the last 5 to 6 decades, substances like multi- phase nature, polymeric metals and solutions do not go with the Newtonian postulate of the linear relationship between shear stress and the rate of shear. Hence, these fluids are considered as non-Newtonian fluids. It is found that these types of fluids exhibit different kinds of behavior varying from the strictness of Newtonian flow behavior. And it safely concludes that the non-Newtonian fluid behavior has a better behavior value while compared to the Newtonian fluid behavior in nature and in technology.

Expressing all the properties of several non- Newtonian fluids in a single constitutive equation are difficult because of the non linearity between the stress and the rate of strain for non-Newtonian fluids. It has gained a research interest of researchers in the study of flow dynamics of non- Newtonian fluids and its variety of applications in engineering, chemical and petroleum industries, construction of paper production, polymer sheet, hot rolling, glass-fabric and wire drawing. The unique characteristics of Casson fluid, one of the non-Newtonian fluids, have wide applications in food processing, in metallurgy, drilling operation and bio-engineering operations, etc. Casson fluid exhibits yield stress. The Casson fluid becomes solid when yield stress, which is greater than sheer stress is applied and such a fluid move when shear stress greater than the yield stress is applied. Jelly, tomato sauce, honey, soup, concentrated fruit juices, etc. are the typical examples of Casson fluid. Another good example of Casson fluid is human blood in which several substances, like protein, fibrinogen, and globulin in aqueous-base plasma, human red blood cells are found and they form a chain like structure, known as aggregates. In engineering applications like thermal design of industrial equipment, dealing with polymeric liquids, molten plastics, and food stuffs or slurries, the flow of non-Newtonian fluids with heat transfer has a very important role to play. It is important to note that the nonlinear relationship between stress and rate of strain is found in non-Newtonian models. A Casson Fluid Model for Multiple Stenosed Artery in the Presence of Magnetic Field is investigated by Rekha and Awasthi (2012). Venkatesan et al. (2013) discussed the Mathematical Analysis of Casson Fluid Model for Blood Rheology in Stenosed Narrow. Bhattacharyya (2013) in his paper studied the steady boundary layer stagnation-point flow of Casson fluid and heat transfer towards a shrinking/stretching sheet. Raju et al. (2015) investigated heat and mass transfer behavior in the Casson fluid past an exponentially permeable stretching surface in the presence of thermal radiation, uniform magnetic field, viscous dissipation, heat source and chemical reaction. Hayat et al.(2012), Dabe et al. (2015) and Butt et al. (2016) have been analyzed Casson fluid flow in various flow situations.

Magneto hydrodynamics (MHD) is the study of the flow of electrically conducting fluids in a magnetic field. Many experimental and theoretical studies on conventional electrically conducting fluids indicate that magnetic field markedly changes their transport and heat transfer characteristics. The study of magneto hydrodynamics has many important applications, and may be used to deal with problems such as cooling of nuclear reactors by liquid sodium and induction flow meter, which depends on the potential difference in the fluid in the direction perpendicular to the motion and to the magnetic field Recently, the application of magneto hydrodynamics in the polymer industry and metallurgy has attracted the attention of many researchers. Many 
applications for the MHD flows of non-Newtonian fluids in a porous medium are encountered in biological systems, heat-storage beds, irrigation problems, process of petroleum, paper, textile and polymer composite industries. Numerous studies have been presented on various aspects of MHD flows of non-Newtonian fluid flows passing through a porous medium. Ullah et al. (2016) studied MHD mixed convection slip flow of Casson fluid over non linearly stretching sheet embedded in a porous medium with chemical reaction, thermal radiation, heat generation/absorption and convective boundary conditions. Casson MHD fluid flow over a vertical plate with a heat source/sink and chemical is studied by Charankumar et al. (2016). Megahed (2015) described MHD viscous Casson fluid flow and heat transfer with secondorder slip velocity and thermal slip over a permeable stretching sheet in the presence of internal heat generation/absorption and thermal radiation. Karmina et al. (2017) analyzed MHD boundary layer flow over a flat plate is governed by the continuity and the Navier Stokes equations for an incompressible viscous fluid.

Viscous dissipation characterizes the degeneration of mechanical energy into the thermal energy. Such phenomenon transpires in all the flow systems. However, for different flow configurations, the characteristics of viscous dissipation are often neglected. It is meaningful just for the systems having larger velocity and velocity gradients respectively. It is for this reason that the viscous dissipation is introduced in the present study. Viscous dissipation is of interest for many applications: significant temperature rises were observed in polymer processing flows such as injection modeling or extrusion at high rates. Medikare et al. (2016) discussed MHD stagnation point flow of a Casson fluid over a non linear stretching sheet with viscous dissipation The Influence of viscous dissipation on the MHD natural convective flow of Casson fluid over an oscillating vertical plate is analyzed by Reddy et al. (2016). Steady, incompressible, laminar flow of an electrically conducting Casson fluid over a melting surface on the upper horizontal paraboloid of revolution in the presence of viscous dissipation and thermal stratification is studied by Ajayi et al. (2016). Recently, Steady magnetohydrodynamic (MHD) flow of Casson liquid in the stretching surface with Joule heating and dissipation effects is investigated by Tamoor et al. (2017).

Thermal radiation impacts might play a major role in controlling heat transfer processes in polymer processing industry. High temperature plasma, cooling of nuclear reactors and power generation systems. The radiative flows of an electrically conducting fluid with high temperature in the presence of magnetic field are e experienced in electrical power era, space vehicle reentry, atomic designing applications and other modern zones. Owing to these applications, the present work deals with a problem of such kind. MHD boundary layer flow of Casson fluid over an exponentially stretching permeable surface with thermal radiation is studied by Mukhopadhyaya et al. (2014). The flow and heat transfer analysis in boundary layer flow of a Casson fluid over an exponentially stretching sheet with combined effects of suction/blowing and thermal radiation are investigated by Pramanik (2014). Pushpalatha et al. (2016) investigated the effects of thermal diffusion and radiation on Casson fluid flow with convective boundary conditions. Casson fluid flow by taking into the account of variable thickness and thermal radiation is analyzed mathematically by Ramesh et al. (2015).

On the other hand, mass transfer is important due to its appearance in many scientific disciplines that involve convective transfer of atoms and molecules. Examples of this phenomenon are evaporation of water, separation of chemicals in distillation processes, natural or artificial sources etc. In addition, mass transfer with chemical reaction has special significance in chemical and hydrometallurgical industries. The impact of magnetohydrodynamic (MHD) and chemical reaction in heat and mass transfer flow has immense importance in many areas of engineering and industries. This phenomenon plays an important role in chemical industry, cooling of nuclear reactors, MHD power generation, MHD pumps, packed-bed catalytic reactor, formation and dispersion of fog, high speed plasma, cosmic jets, enhanced oil recovery, distribution of temperature and moisture over agriculture fields, cooling of nuclear reactors, manufacturing of ceramics, underground energy transport, food processing and cooling towers, etc. Effects of mass transfer on MHD flow of Casson fluid with chemical reaction and suction is studied by Shehzad et al. (2013). Diffusion of chemically reactive species in Casson fluid flow over a stretching surface in porous medium in the presence of a magnetic field is studied by Makande (2015). The boundary layer flow and heat transfer of Casson fluid passing through non linearly permeable stretching surface with chemical reaction in the presence of variable magnetic field is analyzed by Reza et al. (2016). Analytic expression for free convective hydromagnetic boundary layer Casson fluid flow past an oscillating vertical plate embedded through porous medium in the presence of uniform transverse magnetic field, thermal radiation and chemical reaction is investigated by Kataria et al. (2016). Mythili et al. (2015) investigated two dimensional free convective Casson fluid flow over a vertical cone saturated with a porous medium with chemical reaction in the presence of non uniform heat source/sink.

So far no significant contribution has been made on Casson fluid flow toward a stretching sheet with viscous dissipation, thermal radiation and chemical reaction in the presence of a temperature gradient dependent heat sink subject to suction is analyzed. Prescribed heat and mass flux boundary conditions are employed on the porous surface. Similarity transformations are applied to convert the governing non linear partial differential equations of momentum, Energy and species concentration in to nonlinear ordinary differential equations which are then solved analytically. The influence of various physical parameters on velocity, temperature and concentration profiles along with the skin friction coefficient and Nusselt and Sherwood numbers are discussed.

\section{FORMULATION OF THE PROBLEM}

Consider the two - dimensional steady boundary layer flow of a Casson fluid flow over a permeable stretching surface with boundary conditions prescribed heat and mass flux in the presence of temperature gradient dependent heat sink. The effect of viscous dissipation, thermal radiation and chemical reaction on the flow, heat and mass transfer are also taken in to consideration. The Cartesian co-ordinate system is chosen with the direction of motion of the flow along the $\mathrm{x}$-axis and perpendicular to it as the y-axis. The rheological equation of state for an isotropic and incompressible flow of a Casson fluid is given as in Sumalatha and Bandari (2015) by

$$
\tau_{i j}= \begin{cases}2\left(\mu_{B}+\frac{P_{y}}{\sqrt{2 \pi}}\right) e_{i j}, & \pi>\pi_{c} \\ 2\left(\mu_{B}+\frac{P_{y}}{\sqrt{2 \pi_{c}}}\right) e_{i j}, & \pi<\pi_{c}\end{cases}
$$

where $\mu_{B}$ and $P_{y}$ are the plastic dynamic viscosity, yield stress of the fluid respectively. Similarly $\pi$ is the product of the component of deformation rate with itself $\pi=e_{i j} e_{i j}, e_{i j}$ is the $(i, j)$ th component of the deformation rate and $\pi_{c}$ is a critical value of this product based on the non-Newtonian model. Under the above mentioned conditions the governing equations of the problem with boundary conditions are as follows by Maleque and Ghose (2015)

$$
\begin{aligned}
& \frac{\partial u}{\partial x}+\frac{\partial \mathrm{v}}{\partial y}=0 \\
& u \frac{\partial u}{\partial x}+\mathrm{v} \frac{\partial u}{\partial y}=v\left(1+\frac{1}{\beta}\right) \frac{\partial^{2} u}{\partial y^{2}}-\frac{\sigma B_{0}^{2} u}{\rho_{n f}}-\frac{v}{K_{p}} u
\end{aligned}
$$




$$
\begin{gathered}
u \frac{\partial T}{\partial x}+\mathrm{v} \frac{\partial T}{\partial y}=\alpha \frac{\partial^{2} T}{\partial y^{2}}+\frac{\mu}{\rho_{C_{p}}}\left(\begin{array}{r}
1 \\
1+\frac{-}{\beta}
\end{array}\right)\left(\begin{array}{l}
\partial u \\
\frac{\partial u}{\partial y}
\end{array}\right)^{2} \\
-\frac{1}{\rho_{C_{p}} \partial y} \frac{\partial q_{r}}{\partial y} \frac{\partial T}{\partial y}
\end{gathered}
$$$$
u \frac{\partial C}{\partial x}+\mathrm{v} \frac{\partial C}{\partial y}=D \frac{\partial^{2} C}{\partial y^{2}}-k_{1}\left(C-C_{\infty}\right)
$$

Where the term $-Q^{\prime} \frac{\partial T}{\partial y}$ represents the term corresponding to the temperature gradient dependent heat sink and Q $=-$ bQf is the volumetric rate of a linear function of the temperature field [Veena et al. (2011)]. The Rosseland diffusion Approximation for the radiation heat flux term

is $q_{r}=\frac{-16 \sigma^{*}}{3 \alpha^{*}} T_{\infty}^{3} \frac{\partial T}{\partial y}$ [Raptis et al. (2004)] and

$\beta=\mu_{B} \frac{\sqrt{2 \pi_{c}}}{P y}$ is Casson fluid parameter.

The boundary conditions are

$$
\begin{aligned}
& \mathrm{u}=\mathrm{ax}, \mathrm{v}=-\mathrm{V}_{0} \\
& \left.\begin{array}{c}
-K \frac{\partial T}{\partial y}=q_{w}=E_{0} x^{2} \\
-D \frac{\partial D}{\partial y}={ }_{m w}=E_{1} x^{2}
\end{array}\right\} \\
& u=0, \quad v \neq 0 \quad T \rightarrow T_{\infty} \quad C \rightarrow C_{\infty} \quad \text { as } \quad y \rightarrow \infty
\end{aligned}
$$

The following similarity transformations are introduced to transform the nonlinear partial differential equations (1) - (4) into the nonlinear ordinary differential equations as

$$
\begin{aligned}
& \psi=\left(a_{\nu_{f}}\right)^{0.5} x f(\eta), \eta=y\left(\frac{a}{v_{f}}\right)^{0.5}, \mathrm{u}=\frac{\partial \Psi}{\partial y}, \mathrm{v}=-\frac{\partial \Psi}{\partial x}, \\
& \left.\mathrm{~T}-\mathrm{T}_{\infty}=\frac{\mathrm{E} 0 \mathrm{x}^{2}}{\mathrm{~K}} \sqrt{\frac{v}{\mathrm{a}}} \mathrm{g}(\eta)\right\} \\
& \left.\mathrm{C}-\mathrm{C}_{\infty}=\frac{E_{1} \mathrm{x}^{2}}{\mathrm{D}} \sqrt{\frac{\mathrm{v}}{\mathrm{a}} \mathrm{h}(\eta)}\right\}
\end{aligned}
$$

Therefore the resulting non-linear differential equations with boundary conditions are given by

$f^{\prime \prime \prime}\left(1+\frac{1}{\beta}\right)+\left(f f^{\prime \prime}-f^{\prime 2}\right)-\left(\frac{1}{R_{p}}+M^{2}\right) f^{\prime}=0$

$$
g^{\prime \prime}+D(1+Q) f g^{\prime}-2 D f^{\prime} g=-E_{C} D\left(1+\frac{1}{\beta}\right) f^{\prime \prime 2}
$$

$$
h^{\prime \prime}+S c f h^{\prime}-2 S c f^{\prime} h-\gamma S_{c} h=0
$$

$$
\begin{aligned}
& \left.\begin{array}{ll}
f^{\prime}(\eta)=1 & f(\eta)=S \\
g^{\prime}(\eta)=-1 & h^{\prime}(\eta)=-1
\end{array}\right\} \quad \text { at } \eta=0 \\
& f^{\prime}(\eta)=0, f(\eta) \neq 0 \quad g(\eta)=0 \quad h(\eta)=0 \text { as } \eta \rightarrow \infty
\end{aligned}
$$

Where

$D=\frac{3 P_{r} R_{d}}{3 R_{d}+4}, R_{d}=\frac{K \alpha^{*}}{4 \sigma^{*} T_{\infty}^{3}}$ (radiation parameter), $\quad R_{P}=\frac{K p^{a}}{v}$

(Permeability Parameter), $P_{r}=\frac{\mu C_{p}}{K}$ (Prandtl number), $\gamma=\frac{k_{1}}{a}$

(Chemical reaction parameter) $\mathrm{E}_{\mathrm{c}}=\frac{a^{2}}{C_{p} \frac{\mathrm{E}_{0}}{\mathrm{~K}} \sqrt{\frac{v}{\mathrm{a}}}}$ (Eckert number),

$S_{C}=\frac{v}{D}$ (Schmidt number), $S=\frac{V_{0}}{\sqrt{v_{a}}}$ (Suction parameter), Q (heat sink parameter).

\section{SOLUTION OF THE PROBLEM}

The set of nonlinear differential equations (8) - (10) with the boundary conditions constitute a boundary value problem and are solved by analytically using confluent hyper geometric functions. The exact solution of the equation (8) subject to the boundary conditions (11) and (12) is obtained by

$$
\left.\begin{array}{l}
f(\eta)=S+\frac{1-e^{-E \eta}}{E} \\
f^{\prime}(\eta)=e^{-E \eta}
\end{array}\right\}
$$

where $\quad E=\frac{1}{2}\left[S+\sqrt{S^{2}+4 \frac{1}{\left(1+\frac{1}{\beta}\right)}\left(1+M^{2}+R_{p}^{-1}\right)}\right]$ 
In order to solve the Energy equation (9) and the Species Concentration equation (10), new variables $\xi$ and $\varsigma$ are defined respectively as follows

$$
\xi^{-1}=\frac{-E^{2} e^{E \eta}}{D(1+Q)} \zeta^{-1}=\frac{-E^{2} e^{E \eta}}{S_{c}}
$$

Introducing the equation (14) in the equations (9) and (10), the following equations are obtained with the boundary conditions

$$
\begin{aligned}
& \xi \frac{d^{2} g}{d \xi^{2}}+\left[\left(1-K_{1}\right)-\xi\right] \frac{d g}{d \xi}+S_{1} g=-\frac{E_{c} E^{4} \xi}{D(1+Q)^{2}}\left(1+\frac{1}{\beta}\right) \\
& \left.\zeta \frac{d^{2} h}{d \zeta^{2}}+\left[1-K_{3}\right]-\zeta\right] \frac{d h}{d \zeta}-\frac{\gamma S_{c}}{E^{2} \zeta} h=0 \\
& g(\xi=0)=0, \frac{d g}{d \xi}\left(\xi=\frac{-D(1+Q)}{E^{2}}\right)=\frac{-E}{D(1+Q)} \\
& h(\zeta=0)=0, \quad \frac{d h}{d \zeta}\left(\zeta=\frac{-S c}{E^{2}}\right)=\frac{-E}{S c}
\end{aligned}
$$

Where $K_{1}=\frac{D(1+Q)}{E^{2}}\left[E^{2}-R_{p}^{-1}\right] ; S_{1}=\frac{2}{(1+Q)}$,

$K_{3}=\frac{S c}{E^{2}}[1+S E]$

Utilizing the equations (17) and (18) the solution to the equations (15) and (16) can be obtained in terms of confluent hyper geometric function and the same is given as follows

$$
\begin{aligned}
& g(\xi)=-\frac{E_{C} E^{4} \xi^{2}}{D(1+Q) S_{1}\left(S_{1}-K_{1}\right)}\left(1+\frac{1}{\beta}\right)- \\
& \frac{\left[\frac{-\xi}{K_{2}}\right]^{K_{1}}{ }_{1} F_{1}\left[K_{1}-S_{1}, K_{1}+1 ; \xi\right]\left[\frac{E}{D(1+Q)}+\frac{E_{c} E^{2}}{\left(S_{1}-K\right)}\left(1+\frac{1}{\beta}\right)\right]}{\left[\frac{-D(1+Q)}{E^{2}}\right]^{K_{1}-1}\left[K_{11} F_{1}\left[K_{1}-S_{1}, K_{1}+1 ;-K_{2}\right]-K_{2} \frac{K_{1}-S_{1}}{K_{1}+1}{ }_{1} F_{1}\left[K_{1}-S_{1}+1, K_{1}+2 ;-K_{2}\right]\right]}
\end{aligned}
$$

$$
h(\zeta)=\frac{\left[\frac{-\zeta}{K_{4}}\right]^{r}{ }_{1} F_{1}[r-2 ; A+1 ; \zeta]}{E K_{4}\left[\frac{r}{K_{4}} 1 F_{1}\left[r-2, A+1 ;-K_{4}\right]-\frac{r-2}{A+1}\left[l_{1} F_{1}\left[r-1, A+2 ;-K_{4}\right]\right]\right]}
$$

$$
K_{2}=\frac{D(1+Q)}{E^{2}}, K_{4}=\frac{S c}{E^{2}}, r=\frac{A+K_{3}}{2} A=\sqrt{\left[K_{3}^{2}+\frac{4 \gamma S_{c}}{E^{2}}\right]}
$$

The equations (19) and (20) can also be expressed as

$$
\begin{aligned}
& g(\eta)=-\frac{E_{C} D(1+Q) e^{-2 E \eta}}{S_{1}\left(S_{1}-K_{1}\right)}\left(\begin{array}{r}
1 \\
\beta
\end{array}\right)+ \\
& \frac{K_{2} e^{-E_{K_{1}} \eta}{ }_{1} F_{1}\left[K_{1}-S_{1}, K_{1}+1 ;-K_{2} e^{-E \eta}\right]\left[\frac{E}{D(1+Q)}+\frac{E_{c} E^{2}}{\left(S_{1}-K_{1}\right)}\left(1+\frac{1}{\beta}\right)\right]}{\left[\left[K_{1}{ }_{1} F_{1}\left[K_{1}-S_{1}, K_{1}+1 ;-K_{2}\right]-K_{2} \frac{K_{1}-S_{1}}{K_{1}+1}{ }_{1} F_{1}\left[K_{1}-S_{1}+1, K_{1}+2 ;-K_{2}\right]\right]\right]} \\
& h(\eta)=\frac{E K_{4}\left[\frac{r}{K_{4}}{ }_{1} F_{1}\left[r-2, A+1 ;-K_{4}\right]-\frac{r-2}{A+1}\left[{ }_{1} F_{1}\left[r-1, A+2 ;-K_{4}\right]\right]\right]}{1 F_{1}\left[r-2, A+1 ;-K_{4} e^{-E \eta}\right]}
\end{aligned}
$$

The physical quantities of interest are the local skin friction coefficient, Nusselt and Sherwood number, which represent shear stress, rate of heat transfer and rate mass transfer, which are given by

$$
\begin{aligned}
& C_{f}=-\left(1+\frac{1}{\beta}\right) f^{\prime \prime}(0) \\
& N u=\frac{1}{g(0)} \\
& S h=\frac{1}{h(0)} \\
& g(0)=-\frac{E_{C} \quad D(1+Q)}{S_{1}\left(S_{1}-K_{1}\right)}\left(1+\frac{1}{\beta}\right)+ \\
& \left.\frac{K_{2}{ }_{1} F_{1}\left[K_{1}-S_{1}, K_{1}+1 ;-K_{2}\right]\left[\frac{E}{D(1+Q)}+\frac{E_{c} E^{2}}{\left(S_{1}-K_{1}\right)}\left(1+\frac{1}{\beta}\right)\right]}{\left[\left[K_{1}{ }_{1} F_{1}\left[K_{1}-S_{1}, K_{1}+1 ;-K_{2}\right]-K_{2} \frac{K_{1}-S_{1}}{K_{1}+1}{ }_{1} F_{1}\left[K_{1}-S_{1}+1, K_{1}+2 ;-K_{2}\right]\right]\right.}\right] \\
& h(0)=\frac{E K_{4}\left[\frac{r}{K_{4}}{ }_{1} F_{1}\left[r-2, A+1 ;-K_{4}\right]-\frac{r-2}{A+1}\left[F_{1}\left[r-1, A+2 ;-K_{4}\right]\right]\right.}{\left.1 F_{1} \mid r-2, A+1 ;-K_{4}\right]}
\end{aligned}
$$

\section{RESULTS AND DISCUSSION}

The effects of viscous dissipation, thermal radiation and chemical reaction on heat and mass transfer in the presence of a temperature gradient dependent heat sink through a porous medium on the MHD Casson fluid subject to suction have been studied. In order to get a clear insight of the physical problem, the velocity, temperature and concentration have been discussed with the help of graphs by assigning numerical values to the governing parameters encountered in the problem. In the present work the effect of magnetic parameter, suction parameter, Casson parameter, permeability parameter, Prandtl number, radiation parameter, heat sink parameter, Eckert parameter, Schmidt number and chemical reaction parameter on flow, heat and mass transfer characteristics have been analyzed. Also, the local skin-friction coefficient, the local Nusselt and Sherwood number at the surface are computed.

To validate the accuracy of the results, comparisons were made with previously published journals. As shown in Table 1, Table 2 and Table 3 , the results are in nice agreement.

Figs. 1-3 depict the velocity, temperature and species concentration profiles for various values of Casson fluid parameter $\beta$. It is identified that the impact of $\beta$ on the velocity and temperature is decreased and 
concentration is increased. Physically, an increase in the magnitude of $\beta$ implies a decrease in yield stress $P y$ of the Casson fluid and increase in the magnitude of plastic dynamic viscosity $\mu$ в. In view of this, the resistance in the fluid flow is produced. This causes a decrease in momentum and thermal boundary layer thickness. Due to the existence of plastic dynamic viscous provoked retarding force that increases concentration.

The influence of magnetic field $\mathrm{M}$ on velocity, temperature and concentration profiles is illustrated in Figs. 4-6. An increase in the magnetic field causes to produce a Lorentz force which works in the opposite direction of the fluid flow. This force has the tendency to slow down the motion of the flow, create more heat in the fluid and increase the friction between the concentration layers. So the effect of increase in $\mathrm{M}$ is to decrease the velocity and increase the temperature and concentration.

Table 1 Comparison of Skin friction coefficient with the previously reported work $(\mathrm{Rp}=0 \& \mathrm{~S}=0)$

\begin{tabular}{|c|c|c|c|}
\hline $\mathrm{M}$ & $\beta$ & Present Result & $\begin{array}{l}\text { Gireesha } \text { et al. } \\
(2015)\end{array}$ \\
\hline & 0.8 & 1.677050 & 1.67712 \\
0.25 & 1.4 & 1.463850 & 1.46386 \\
& 2.0 & 1.369306 & 1.36931 \\
& 3.0 & 1.290994 & 1.29099 \\
\hline
\end{tabular}

Table 2 Comparison of $g(0)$ with the previously reported work $\left(\mathrm{Rp}=100, \mathrm{~S}=0, \mathrm{Q}=0, \mathrm{Ec}=0, \mathrm{R}_{\mathrm{d}}=0, \beta \rightarrow \infty\right)$

\begin{tabular}{|c|c|c|c|}
\hline M & Pr & Present Result & $\begin{array}{l}\text { Abdul } \\
\text { Hakeem } \text { et al. } \\
\text { al(2014) }\end{array}$ \\
\hline & 1 & 0.822522 & 0.82252 \\
1 & 5 & 0.311797 & 0.31179 \\
& 10 & 0.213209 & 0.21321 \\
\hline
\end{tabular}

Table 3 Comparison of $\mathrm{h}(0)$ with the previously reported work $\left(\mathrm{Rp}=100, \mathrm{~S}_{\mathrm{c}}=0.62, \gamma=0, \& \beta \rightarrow \infty\right)$

\begin{tabular}{|l|c|c|l|}
\hline $\mathrm{M}$ & $\mathrm{S}$ & Present Result & $\begin{array}{l}\text { Anjalidevi } \text { et } \\
\text { al. (2013) }\end{array}$ \\
\hline & 0.5 & 1.132885 & 1.1328 \\
& 1.0 & 0.900560 & 0.900561 \\
& 1.5 & 0.737860 & 0.737861 \\
& 2.0 & 0.619962 & 0.619963 \\
& 2.5 & 0.531762 & 0.531763 \\
\hline
\end{tabular}

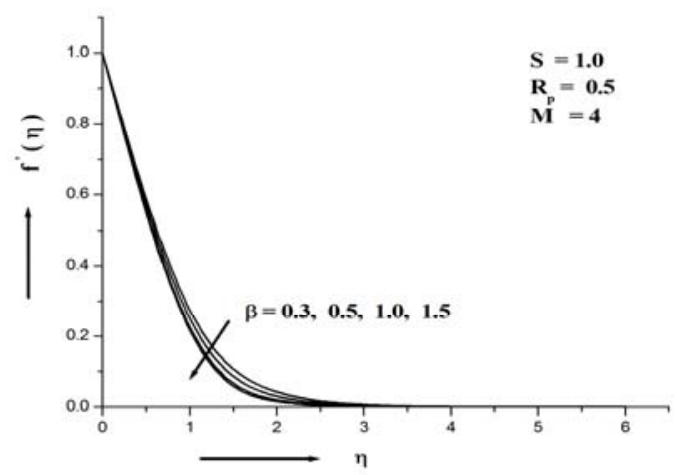

Fig. 1 Velocity profiles for various values of $\beta$

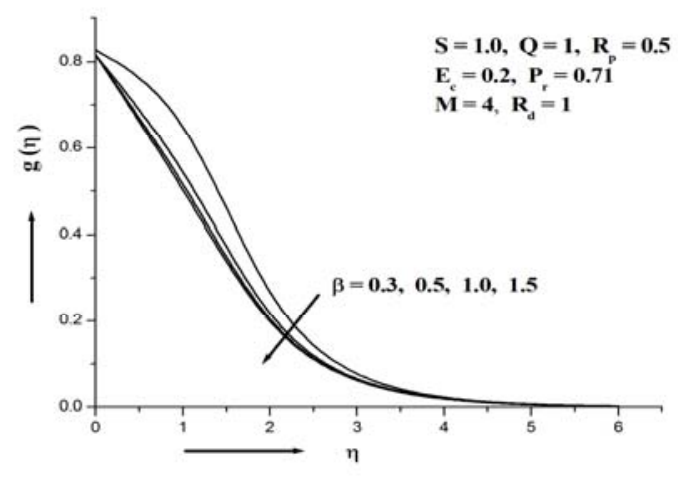

Fig. 2 Temperature profiles for various values of $\beta$

The influence of Suction parameter S on velocity, temperature and species concentration distribution is depicted in Figs. 7-9. It is explored that the effect of $\mathrm{S}$ on velocity, temperature and concentration is decreased. While increasing the suction parameter the fluid moves towards the porous surface. This causes to reduce the thickness of the momentum, thermal and concentration boundary layer thickness.

Figs. 10-12 indicate the impact of Permeability parameter Rp on velocity temperature and concentration. From these figures it is found that the increase of $\mathrm{Rp}$ results in the enhancement of velocity distribution and reduction of temperature and species concentration distributions. The increase of permeability of the porous medium which increase the size of the porosity and hence the fluid can freely move through the boundary layer. This leads to raise the velocity, thermal and concentration boundary layer region.

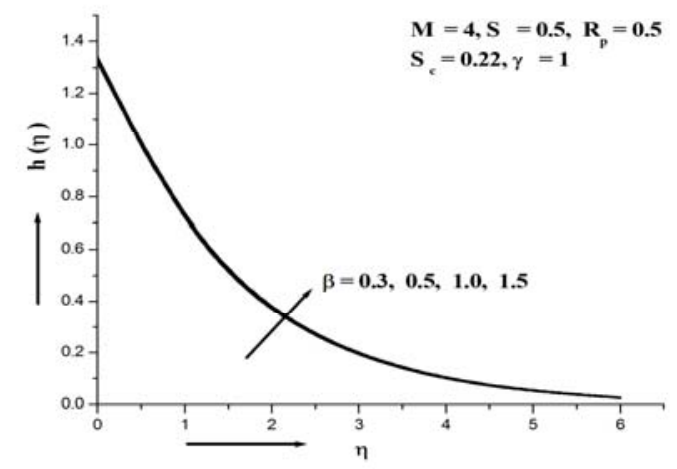

Fig. 3 Concentration profiles for various values of $\beta$

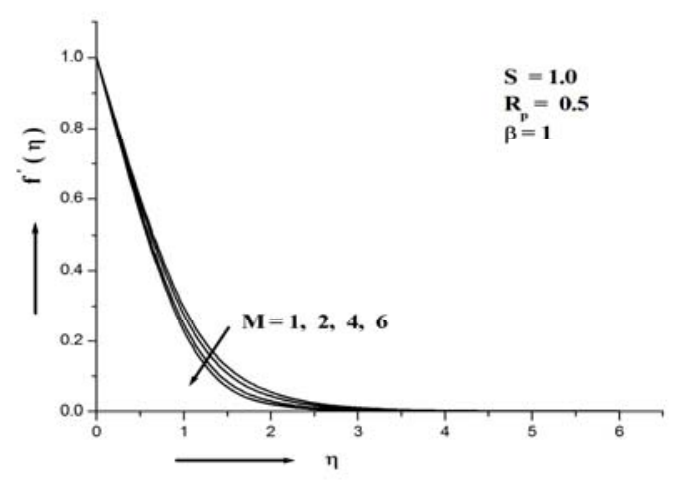

Fig. 4 Velocity profiles for various values of $M$ 


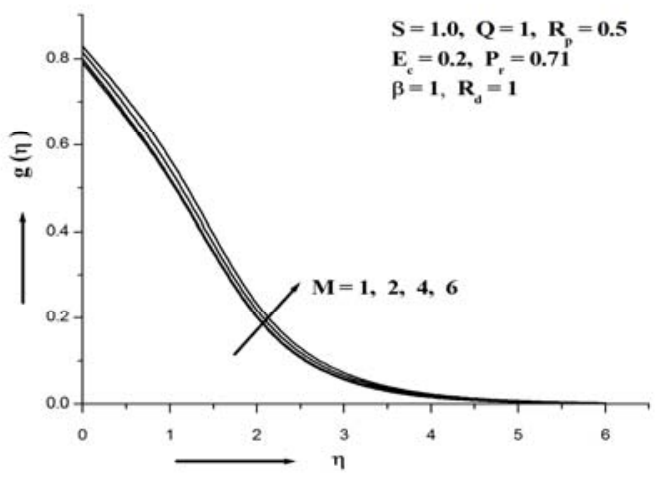

Fig. 5 Temperature profiles for various values of $M$

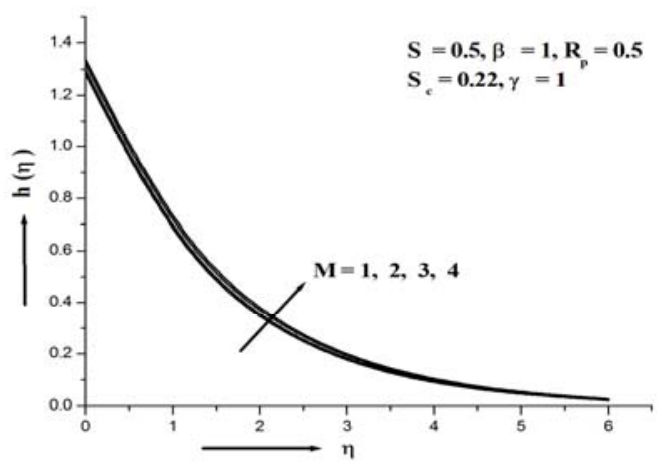

Fig. 6 Concentration profiles for various values of $M$

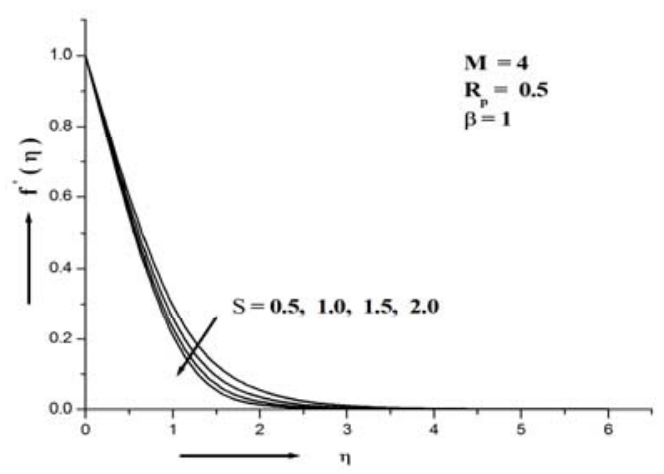

Fig. 7 Velocity profiles for various values of $\mathrm{S}$

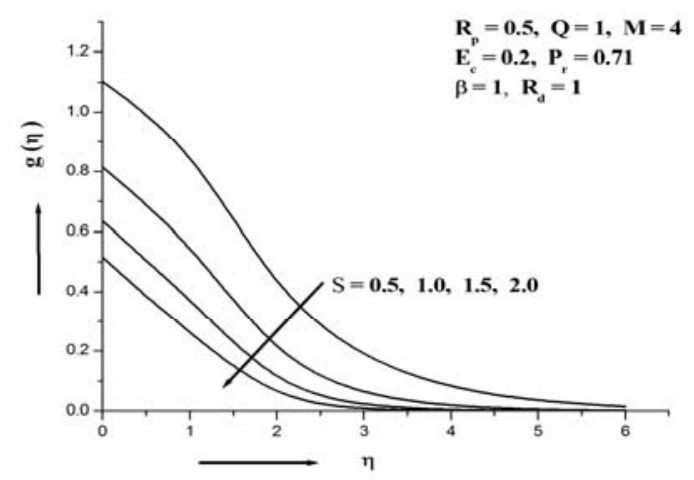

Fig. 8 Temperature profiles for various values of $S$

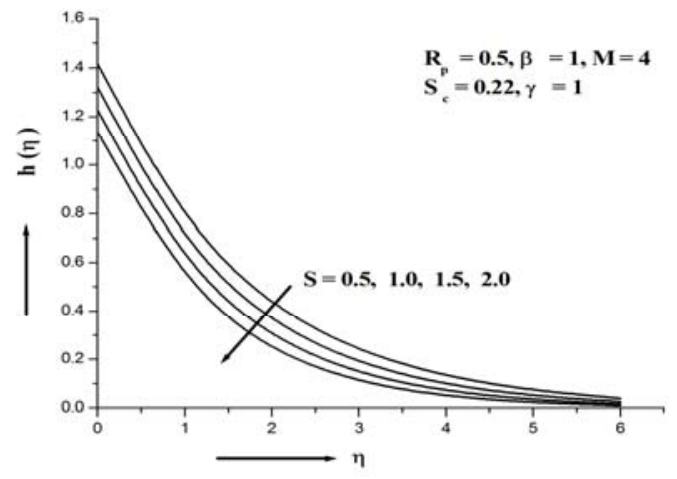

Fig. 9 Concentration profiles for various values of $\mathrm{S}$

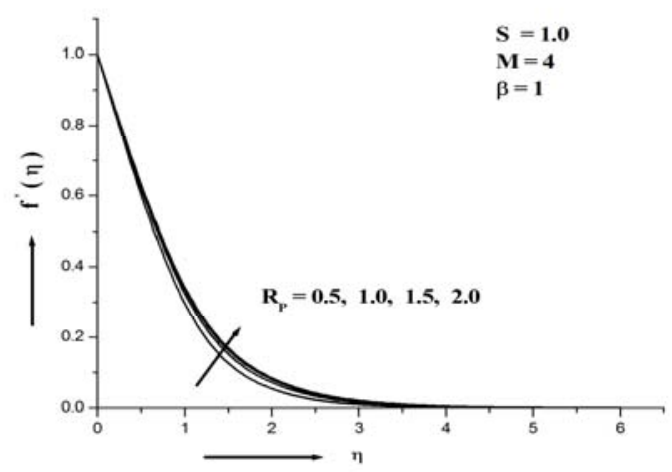

Fig. 10 Velocity profiles for various values of $R_{p}$

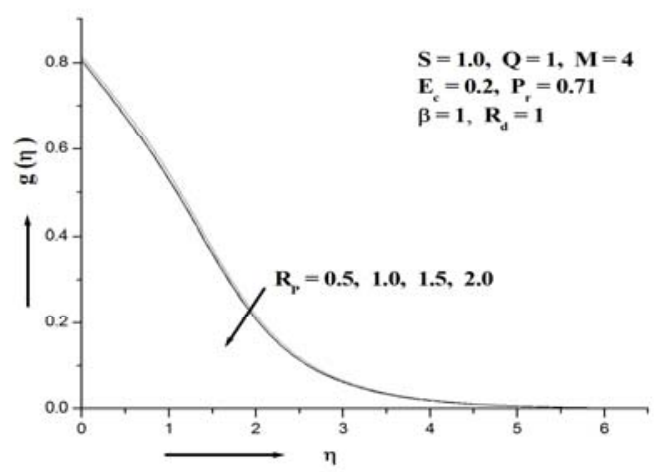

Fig. 11 Temperature profiles for various values of $R_{p}$

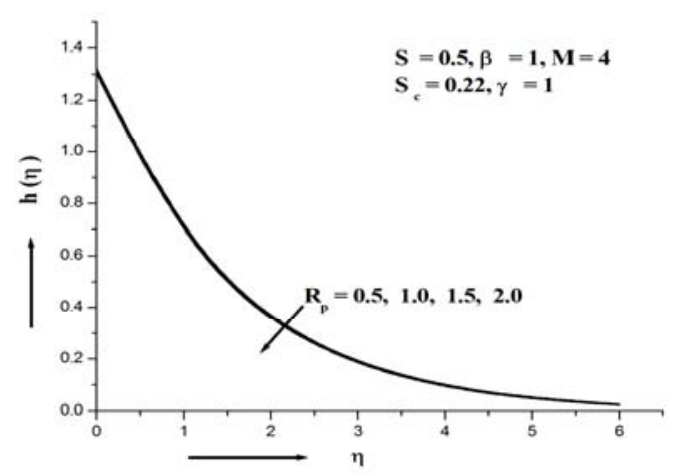

Fig. 12 Concentration profiles for various values of $R_{p}$ 
Fig. 13 exhibits the effect of Prandtl number $\operatorname{Pr}$ on the temperature profile. The ratio between kinematic viscosity and thermal diffusivity is defined as the Prandtl number. It is seen that the temperature within the fluid is sharply decreased when Pr is increased. When the Prandtl number is increased, thermal diffusivity decreases, which reduce the thermal boundary layer thickness.

The temperature profile is depicted in Fig. 14 for different values of Heat sink parameter Q. It is observed that while increasing the heat sink parameter $\mathrm{Q}$ the temperature is decreased. As the heat sink parameter is increased more heat is transferred from the fluid to the surface and thus the temperature decreases.

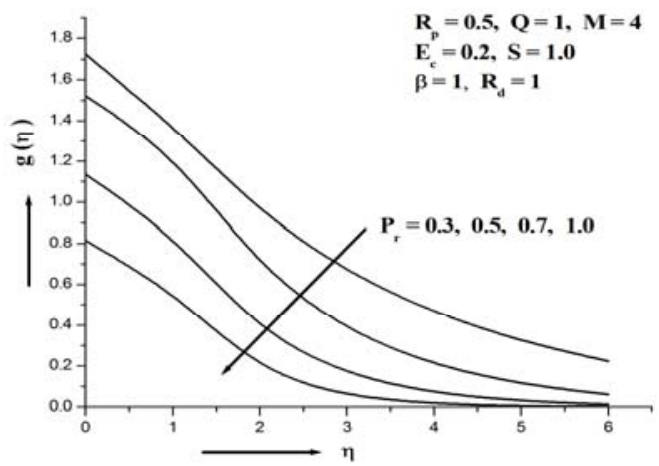

Fig. 13 Temperature profiles for various values of $\mathrm{P}_{\mathrm{r}}$

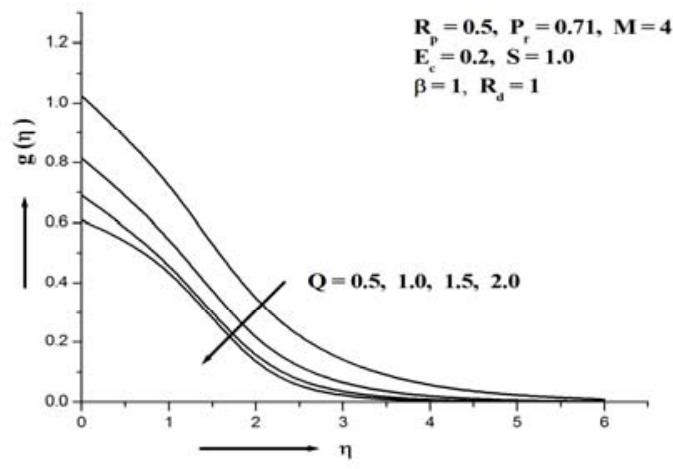

Fig. 14 Temperature profiles for various values of $Q$

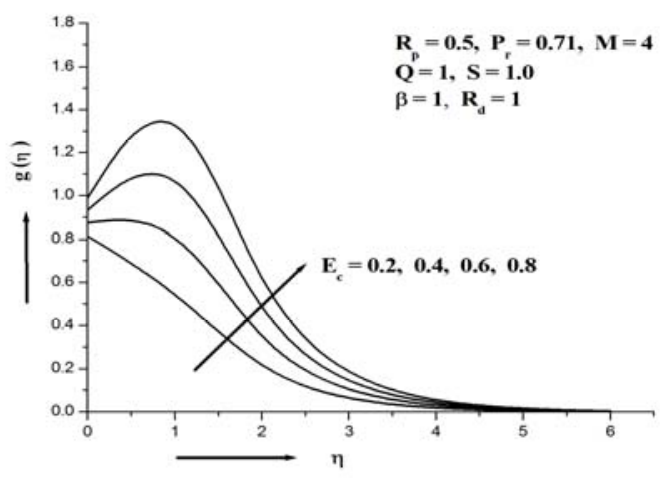

Fig. 15 Temperature profiles for various values of Ec

Fig. 15 signifies the influence of Eckert number Ec on the temperature profile. It is noted that the temperature is increased for improved values of Ec. This is accordance with the fact that the heat generated within the fluid due to the viscous dissipation. The Eckert number signifies the ratio between the flow of kinetic energy and enthalpy difference of the boundary layer. It represents the conversion of kinetic energy into internal energy by work done against the viscous fluid stresses. Greater viscous dissipative heat causes an increase in the fluid temperature. Temperature profiles for different value of radiation parameter $\mathrm{Rd}$ is depicted in Fig. 16. The figure reveals that the effect of $\mathrm{Rd}$ on temperature is decreased. An increase in the thermal radiation parameter corresponds to an increase in the rate of heat transfer caused a reduction in temperature.

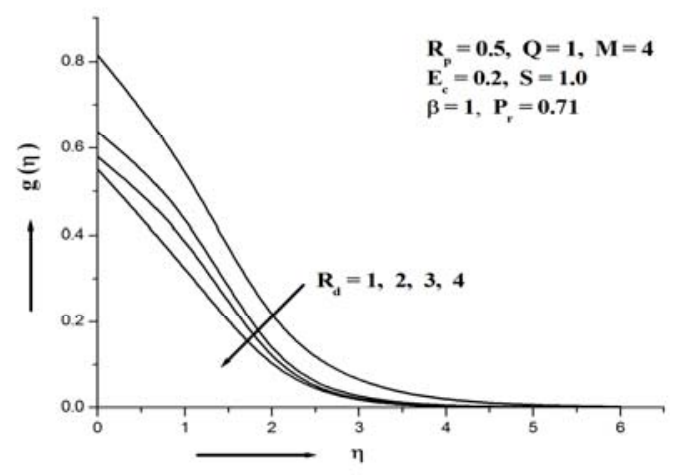

Fig. 16 Temperature profiles for various values of $R_{d}$

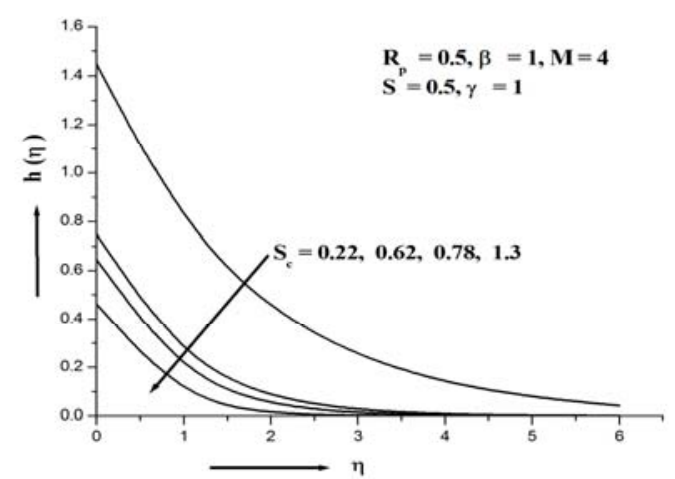

Fig. 17 Concentration profiles for various values of $\mathrm{S}_{c}$

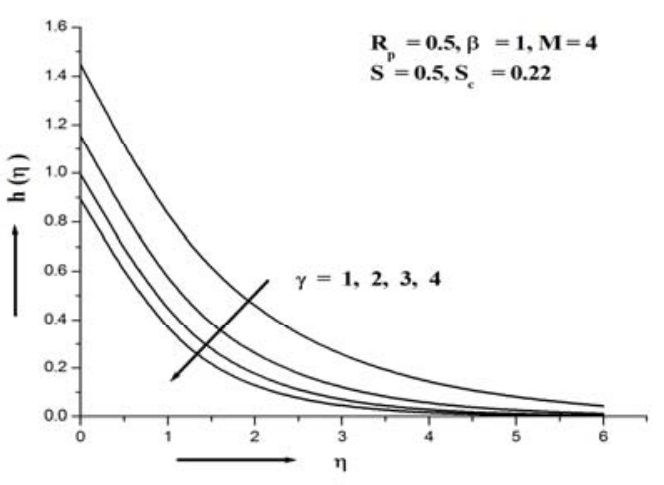

Fig. 18 Concentration profiles for various values of $\gamma$

Fig. 17 demonstrates the effect of Schmidt number Sc on the species concentration profile. It is viewed that concentration is decreased for improved values of Sc. Schmidt number is a ratio between momentum diffusivity and mass diffusivity. If Schmidt number increases the mass diffusivity decreases, this leads to reduce the concentration boundary layer thickness. 


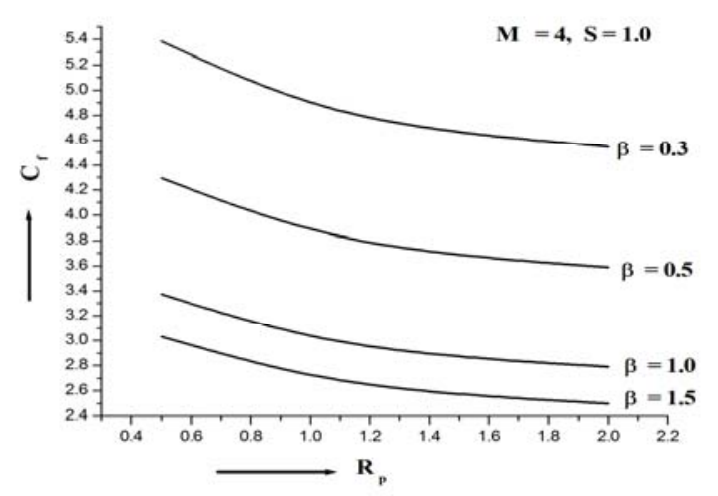

Fig. 19 Skin friction Coefficient for various $\beta$

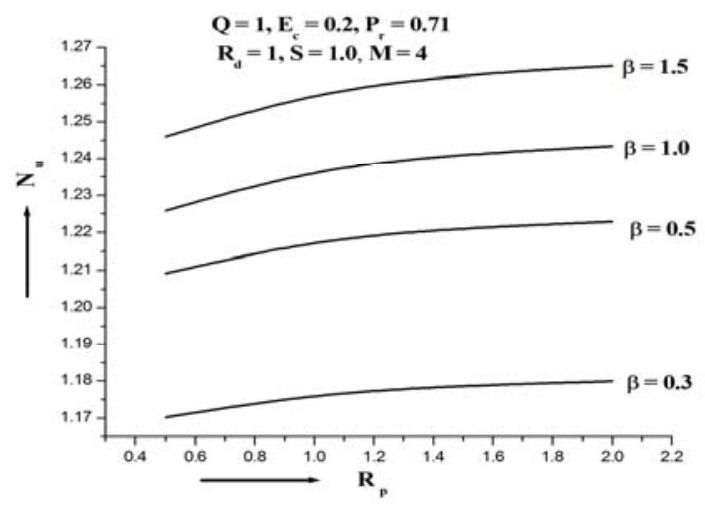

Fig. 20 Nusselt number for various $\beta$

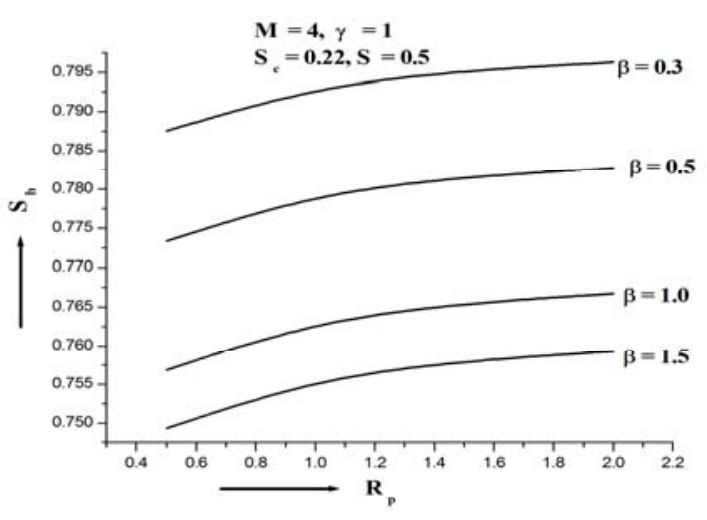

Fig. 21 Sherwood number for various $\beta$

Fig. 18 portrays the species concentration for various values of chemical reaction parameter $\gamma$. As $\gamma$ increases, the concentration decreases. An increase in the chemical reaction rate parameter caused a reduction in concentration.

The effect of $\beta$ on skin friction coefficient is explored in Figs. 19. The Skin friction coefficient is a ratio between shear/yield stress to characteristic dynamic pressure. It is revealed from the figures that the skin friction coefficient decreases with increasing value of $\beta$. This is caused by shear/yield decreases for enhanced values of $\beta$.

The influence of parameter $\beta$ on the Nusselt number is shown in Fig. 20 . From this figure, it confirmed that the Nusselt number is increasing for improved values of $\beta$. As increase in $\beta$, heat transfer coefficient is increased.
Sherwood number of various values of $\beta$ is portrayed in Fig. 21. From this figure, it is concluded that the values of the Sherwood number decrease upon increasing $\beta$. This is due to the fact that mass diffusivity decreases while increase in $\beta$.

\section{CONCLUSIONS}

The analysis of MHD Casson flow with viscous dissipation, thermal radiation and chemical reaction in the presence of a temperature gradient dependent heat sink subject to suction has been carried out through this paper. In general, all the physical parameters affect the velocity, temperature, species concentration distribution, skin friction coefficient, Nusselt and Sherwood numbers of the MHD Casson fluid.

1) The velocity increases with the increase of Permeability parameter and decreases with increasing values of Casson Fluid Parameter, Suction Parameter and Magnetic Parameter.

2) The temperature distribution increases while increasing values of Magnetic parameter, Eckert number and decreases for improving the values of Casson fluid parameter, Suction Parameter, Permeability Parameter, Prandtl Number, Heat Sink Parameter and Radiation Parameter.

3) The species concentration distribution increases with increasing values of the Casson fluid parameter and Magnetic parameter and decreases for enhancing the values of Suction Parameter, Permeability Parameter, Schmidt Number and Chemical Reaction Parameter.

4) The skin friction coefficient, Nusselt and Sherwood number of the different values of Casson fluid parameter are analyzed.

\section{NOMENCLATURE}

a dimensional constant

C species concentration of the fluid $\left(\mathrm{kg} / \mathrm{m}^{3}\right)$

$\mathrm{C}_{\mathrm{p}} \quad$ specific heat at the surface $\mathrm{J} / \mathrm{kg} . \mathrm{K}$

$\mathrm{C}_{\mathrm{W}} \quad$ species concentration at the surface $\left(\mathrm{kg} / \mathrm{m}^{3}\right)$

$\mathrm{C}_{\infty} \quad$ species concentration away from the surface $\left(\mathrm{kg} / \mathrm{m}^{3}\right)$

D mass diffusivity coefficient $\left(\mathrm{m}^{2} / \mathrm{s}\right)$

$\mathrm{E}_{0}, \mathrm{E}_{1} \quad$ positive constants

$\mathrm{K}$ thermal conductivity (W/mK)

$\mathrm{K}_{\mathrm{p}} \quad$ permeability of the medium $\left(\mathrm{m}^{2}\right)$

$\mathrm{m}_{\mathrm{w}} \quad$ rate of mass transfer $\left(\mathrm{Kg} / \mathrm{m}^{2} \mathrm{~s}\right)$

$\mathrm{T}$ temperature of the dynamic fluid $(\mathrm{K})$

$\mathrm{T}_{\mathrm{W}} \quad$ temperature at the surface $(\mathrm{K})$

$\mathrm{T}_{\infty} \quad$ temperature away from the surface.

k1 dimensional chemical parameter

Q volumetric rate of heat absorption

$\mathrm{q} r \quad$ radiative heat flux $\left(\mathrm{kW} / \mathrm{m}^{2}\right)$

$\mathrm{q}_{\mathrm{w}} \quad$ rate of heat transfer $\left(\mathrm{kW} / \mathrm{m}^{2}\right)$

$\mathrm{V}_{0} \quad$ constant suction velocity $(\mathrm{m} / \mathrm{s})$

\section{Greek Symbols}

$v \quad$ kinematic coefficient of viscosity $\left(\mathrm{m}^{2} / \mathrm{s}\right)$

$\mu \quad$ coefficient of viscosity(Pascal)

$\rho \quad$ density $\left(\mathrm{kg} / \mathrm{m}^{3}\right)$

$\sigma^{*} \quad$ Stefan-Boltzmann constant $\left(\mathrm{W} / \mathrm{m}^{2} \cdot \mathrm{K}^{4}\right)$

$\alpha^{*} \quad$ mean absorption coefficient of the fluid. 


\section{REFERENCES}

Abdul Hakeem, A.K., Kalaivanan, R., Vishnu Ganesh, N. and Ganga, B., 2014, "Effect of Partial Slip on Hydro Magnetic Flow over a Porous Stretching Sheet with Non-Uniform Heat Source/Sink, Thermal Radiation and Wall Mass Transfer," Ain Shams Engineering Journal, 5(3), 913-922.

http://dx.doi.org/10.1016/j.asej.2014.02.006

Ajayi, T.M., Omowaye, A.J. and Animasaun, I.L., 2017, "Viscous Dissipation Effects on the Motion of Casson Fluid over an Upper Horizontal Thermally Stratified Melting Surface of a Paraboloid of Revolution: Boundary Layer Analysis," Journal of Applied Mathematics, Volume 2017 (2017), Article ID 1697135.

https://doi.org/10.1155/2017/1697135

Anjali Devi, S.P., and M.Kayalvizhi, M., 2013, "Nonlinear Hydro Magnetic Flow with Radiation and Heat Source over a Stretching Surface with Prescribed Heat and Mass Flux Embedded in a Porous Medium,"Journal of Applied fluid Dynamics, 6 (2), 157 -165. https://www.researchgate.net/publication/274701626

Bhattacharyya, K., 2013, "Boundary Layer Stagnation-Point Flow of Casson Fluid and Heat Transfer towards a Shrinking/Stretching Sheet," Frontiers in Heat and Mass Transfer (FHMT), 4, 023003. http://dx.doi.org/10.5098/hmt.v4.2.3003

Butt, A.S., Tufail, M.N., and Asif Ali., 2016, “Three-Dimensional Flow of a Magnetohydrodynamic Casson fluid over an Unsteady Stretching Sheet Embedded into a Porous Medium," Journal of Applied Mechanics and Technical Physics, 57(2), 283-292.

https://doi.org/10.1134/S0021894416020115

Charankumar, G., Dharmaiah, G., Balamurugan, K.S. and Vedavathi, N., 2016, "Chemical Reaction and Soret Effects on Casson MHD Fluid Flow over a Vertical Plate,” Int. J. Chem. Sci.: 14 (1), 213-221. https://www.researchgate.net/publication/302215685

El-Dabe, N.T., Ghaly, A.Y., Rizkallah, R.R., Ewis, K.M. and Al-Bareda, A.S., 2015, "Numerical Solution of MHD Boundary Layer Flow of NonNewtonian Casson Fluid on a Moving Wedge with Heat and Mass Transfer and Induced Magnetic Field, "Journal of Applied Mathematics and physics," 3, 649-663.

http://dx.doi.org/10.4236/jamp.2015.36078

Gilbert Makanda., Sachin Shaw. and Precious Sibanda., 2015, "Effects of Radiation on MHD Free Convection of a Casson Fluid from a Horizontal Circular Cylinder with Partial Slip in Non-Darcy Porous Medium with Viscous Dissipation," Boundary Value Problems, 75. https://doi.org/10.1186/s13661-015-0333-5

Gireesha., B.J., Mahanthesh, B. and Rashidi, M.M., 2015, "MHD Boundary Layer Heat and Mass Transfer of a Chemically Reacting Casson Fluid over a Permeable Stretching Surface with Non-Uniform Heat Source/Sink," Int. J. Industrial Mathematics , 7(3), Article ID IJIM-00628, 247-260.

http://ijim.srbiau.ac.ir/article 7192

Hayat, T., Shehzad, S.A. and Alsaedi, A., 2012, "Soret and Dufour Effects in Magnetohydrodynamic (MHD) flow of Casson fluid," Appl. Math. Mech.-Eng., 33, 1301-1312.

https://doi.org/10.1007/s10483-012-1623-6.

Jithender Reddy, G., Srinivasa Raju, R. and. Anand Rao, J., 2017, "Influence of Viscous Dissipation on Unsteady MHD Natural Convective Flow of Casson Fluid over an Oscillating Vertical Plate Via FEM,"Ain Shams Engineering Journal. http://dx.doi.org/10.1016/j.asej.2016.10.012
Maleque,Kh.A. and Ghose, P.K., 2015, "Non-Newtonian Casson Fluid Heat and Mass Transfer Flow and Viscous Dissipation with a Binary Chemical Reaction," The AIUB Journal of Science and Engineering, 14(1), 201-214.

https://orp.aiub.edu/ajse-vol-14-no-1-august-2015

Megahed, A.M., 2015, “ MHD Viscous Casson Fluid Flow And Heat Transfer with Second-Order Slip Velocity and Thermal Slip over a Permeable Stretching Sheet in the Presence of Internal Heat Generation/Absorption and Thermal Radiation," The European Physical Journal Plus, 130 (4).

https://doi.org/10.1140/epjp/i2015-15081-9

Monica Medikar., Sucharitha Joga. and Kishore Kumar Chidem., 2016, "MHD Stagnation Point Flow of a Casson Fluid over a Nonlinearly Stretching Sheet with Viscous Dissipation," American Journal of Computational Mathematics, 6, 37-48.

http://dx.doi.org/10.4236/ajcm.2016.61005

Motahar Reza., Rajni Chahal. and Neha Sharma., 2016, "Radiation Effect on MHD Casson Fluid Flow over a Power-Law Stretching Sheet with Chemical Reaction," World Academy of Science, Engineering and Technology International Journal of Chemical, Molecular, Nuclear, Materials and Metallurgical Engineering, 10, 585-590.

http://waset.org/publications/10004434

Mythili, D., Sivaraj, R., Rashidi, M.M. and Yang, Z., 2015, "Casson Fluid Flow over a Vertical Cone with Non-Uniform Heat Source/Sink and High Order Chemical Reaction", Journal of Naval Architecture and Marine Engineering, 15, 125-136.

http://dx.doi.org/10.3329/jname.v12i2.25269

Karmina, K. Ali., Hajar, F. Ismael., Bewar, A. Mahmood. and Majeed, A. Yousif., 2017, “ MHD Casson fluid with heat transfer in a liquid film over unsteady stretching plate," International Journal of Advanced and Applied Sciences, 4, $55-58$.

https://doi.org/10.21833/ijaas.2017.01.008

Kataria, H.R. and Patel, H.R., 2016, "Radiation and Chemical Reaction Effects on MHD Casson Fluid Flow Past an Oscillating Vertical Plate Embedded in Porous Medium," Alexandria Engineering Journal, 55, 583-595.

http://dx.doi.org/10.1016/j.aej.2016.01.019

Raju, C.S.K., Sandeep, N., Sugunamma, V., Jayachandra Babu, M. and Ramana Reddy, J.V., 2016, "Heat And Mass Transfer in Magnetohydrodynamic Casson Fluid over an Exponentially Permeable Stretching Surface," Engineering Science and Technology, an International Journal, 19, 45-52.

http://dx.doi.org/10.1016/j.jestch.2015.05.010

Ramesh, G.K., Prasannakumara, B.C., Gireesha, B.J. and Rashidi, M.M., 2016, "Casson Fluid Flow near the Stagnation Point over a Stretching Sheet with Variable Thickness and Radiation," Journal of Applied Fluid Mechanics, 9, 1115-1122.

http://dx.doi: 10.18869/acadpub. jafm. 68.228.24584

Raptis, A., Perdikis, C. and Takhar, H.S., 2004, "Effect of Thermal Radiation on MHD Flow," Appl Math Comput. 153, 645-49. https://doi.org/10.1016/S0096-3003(03)00657-X

Rekha Bali. and Usha Awasthi., 2012, "A Casson Fluid Model for Multiple Stenosed Artery in the Presence of Magnetic Field," Applied Mathematics, 3, 436-441.

http://dx.doi.org/10.4236/am.2012.35066

Pramanik, S., 2014, "Casson Fluid Flow and Heat Transfer Past an Exponentially Porous Stretching Surface in Presence of Thermal 
Radiation," Ain Shams Engineering Journal, 5, 205-212. http://dx.doi.org/10.1016/j.asej.2013.05.003

Pushpalatha, K., Sugunamma, V., Ramana Reddy, J.V. and Sandeep, N., 2016, “ Heat and Mass Transfer in Unsteady MHD Casson Fluid Flow with Convective Boundary Conditions," International Journal of Advanced Science and Technology, 91, 19-38. http://dx.doi.org/10.14257/ijast.2016.91.03

Shehzad, S.A., Hayat, T., Qasim, M. and Asghar, S., 2103, "Effects of Mass Transfer on MHD Flow of Casson Fluid with Chemical Reaction and Suction," Brazilian Journal of Chemical Engineering, 30, 187 - 195. http://dx.doi.org/10.1590/S0104-66322013000100020

Sumalatha, S. and Bandari, S., 2015, "Effects of Radiations and Heat Source/Sink on a Casson Fluid Flow over Nonlinear Stretching Sheet," World Journal of Mechanics, 5, 257-265. http://dx.doi: 10.4236/wjm.2015.512024

Swati Mukhopadhyay., Iswar Chandra Moindala. and Tasawar Hayat., 2014, "MHD Boundary Layer Flow of Casson Fluid Passing Through an Exponentially Stretching Permeable Surface with Thermal Radiation," Chin. Phys. B, 23, 104701.

http://dx.doi.org/10.1088/1674-1056/23/10/104701
Tamoor, M., Waqas, M., Ijaz Khan, M., Ahmed Alsaedi. and Hayat, T., 2017, "Magnetohydrodynamic Flow of Casson Fluid over a Stretching Cylinder," Results in Physics., 7, 498-502.

http://dx.doi.org/10.1016/j.rinp.2017.01.005

Ullah, I., Bhattacharyya, K., Sharidan Shafie. and Ilyas Khan., 2016, "Unsteady MHD Mixed Convection Slip Flow of Casson Fluid over Nonlinearly Stretching Sheet Embedded in a Porous Medium with Chemical Reaction, Thermal Radiation, Heat Generation/Absorption and Convective Boundary Conditions," PLOS ONE, 24, 0165348. http://dx.doi:10.1371/journal.pone.0165348

Veena, P.H., Pravin,V.K. and Ashok Kumar, K., 2011, "MHD Flow and Heat Transfer with Temperature Dependent Heat Sink in a Porous Medium Past a Stretching Surface,'International Journal of Chemical Engineering, 4, 133-146.

http://serialsjournals.com/serialjournalmanager/pdf/1332136069.pdf

Venkatesan, J., Sankar,D.S., Hemalatha, K. and Yazariah Yatim., 2013, "Mathematical Analysis of Casson Fluid Model for Blood Rheology in Stenosed Narrow Arteries," Journal of Applied Mathematics, Article ID 583809.

http://dx.doi.org/10.1155/2013/583809 